6. Voir à ce sujet : DoRner W. - L'ensilage du fourrage vert. Annuaire agricole de la Suisse, 1927, p. 295.

7. RUTGERS F. - Bericht über Versuche mit elektrischer Grünfutterkonservierung auf dem Gute Bocken bei Horgen. 1925, Maschinenfabrik Oerlikon.

8. Kuchier L. F. - Die ze:tgemässe Grünfutterkonservierung 1926, Datterer, Freising-München.

9. Burri R. - Zum Wiederaufleben der Pressfutterfrage. Schweiz Milchzeitung, 1918, Nr. 18.

10. DorNer W. - Beobachtungen über das Verhalten der Sporen und vegetativen Formen von Bac. amylobacter A.M. et Bredemann bei Nachweis- und Reinzuchtversuchen. Landw. Jahrbuch der Schweiz, 1924.

11. Kürsteiner J. - Ist Milch aus Ställen, in welchen den Kühen konserviertes Gras, sog. Süssgrünfutter, verabreicht wird, für die Emmentalerkäserei tauglich? Schweiz. Zentralblatt für Milchwirtschaft, 1918, Nos. 29-30.

12. Burri R., Staub W. und Hoнl J. - Süssgrünfutter und Buttersäurebazillen. Schweiz. Milehzeitung 1919, Nos. 78-83 und Schweiz. Zentralblatt für Milchwirtschaft, 1919, Nos. 40-43.

13. KÜRSTEINER J. - Ueber eine durch nachträgliche Blähung verursachte schwere Käserei-Betriebsstörung. Schweiz. Milchzeitung 1919, Nr. 3.

14. KürRsteiner J. - Zur Frage der Käsereitauglichkeit der Süssgrünfuttermilch. Schweiz. Milchzeitung 1919, Nos. 72-75 und Schweiz. Zentralblatt für Milchwirtschaft, 1919, Nos: 42-43.

15. Kürsteiner J., Staub W. und Dorner W. - Ist besonders reinlich gemolkene, aus konserviertem Gras erzeugte Milch für die Emmentalerkäserei tauglich ? Schweiz. Milchzeitung, 1922, Nr. 72.

16. Kürsteiner J. und StaUB W. - Ein weiterer Beitrag zur Abklärung der Süssgrünfutterfrage. Schweiz. Zentralblatt für Milchwirtschaft, 1. Dec. 1921.

17. Kǘrsteiner J. - Neuerdings aufgetretene, durch den Blähungserreger des Süssgrünfutters verursachte Käsereibetriebsstörungen, Schweiz. Zentral blatt für Milchwirtschaft, 1920.

18. Kürsteiner J. - Die heutige Graskonservierung, eine grosse Gefahr für die Emmentalerkäserei. Schweiz. Milchzeitung, 1922, Nos. 29-33.

\title{
L'ACTION DES HALOGËNES SUR LE LAIT ET SUR SES CONSTITUANTS
}

\author{
Par le Dr ALb-.J.-J. VANDEVELDE,
}

Professeur à l'Institut agronomique et à l'Université de l'Etat à Gand.

Les halogènes sont susceptibles d'agir sur le lait et sur ses constituants, comme antiseptiques, mais ils peuvent aussi agir chimiquement. A la suite de mes recherches sur les combinaisons halogénées des protides (1), j'ai été amené à étudier l'action des halogènes sur le lait et sur

(1) Protéines halogénées : I. Bromovalbumine, Rec. trav. chim. Pays-Bas, 1924, 43, 158. II. Bromof эptone, ibid., 1924, 43, 326. III. Bromogluten, ibid., 1924, 43, 706. IV. Bromofibrine, ibid., 1925, 44, 224. V. Bromocaséine, ibid., 1925, 44, 900. Halogenated proteins. VI. Chlorocasein, ibid., 1926, 45, 825. VII. Chlorofibrin, ibid., 1927, 46, 133. VIII. Chlorogluten, ibid,, 1927, 46, 590. IX. Chlorovoprotein, ibid., 1928, 47, 458. 
ses constituants : le chlore et le brome provoquent une rapide coagulation; l'iode à l'état pulvérulent peut stériliser le lait sans coagulation de la caséine. Il se produit en même temps des combinaisons halogénées de la caséine; le lactose est oxydé partiellement ; les teneurs en extrait, cendres et acide sont augmentées; seule la graisse semble ne subir aucune influence sensible.

\section{A. Action du chlore.}

Mrnet (1) a étudié l'action du chlorure de chaux, surtout en vue de la stérilisation du lait ; des solutions à $4 \%$ de chlorure de chaux titrant $29.9 \%$ de chlore actif (ou pour mieux dire $6.7 \%$ d'oxygène actif (2)) n'ont guère donné de résultats favorables, même en quantité de $3 \mathrm{~cm}^{3}$ de solution pour $100 \mathrm{~cm}^{3}$ de lait. Nul doute, en effet, que le chlorure de chaux oxyde rapidement certains des constituants du lait, et se trouve par suite rapidement décomposé.

Le gaz chlore provoque à froid une rapide coagulation de la caséine ; le précipité est une caséine chlorée contenant $12.2 \%$ d'azote et $11.6 \%$ de chlore.

Analyse du précipité après filtration, lavage et dessication : substance : 0 gr., 1634 ; azote (Kjeldahl) : 0 gr., 0199, soit 12.2\%. Substance: 0 gr., 0183 ; chlore (méthode ter Meulen) : 0 gr., 00213, soit 11.6\%.

Dans mes recherches sur la chlorocaséine (3) j'ai obtenu les produits suivants :

dans un milieu de tétrachlorure de earbone : $10,5 \% \mathrm{~N}$. et $32 \% \mathrm{Cl}$. en présence d'eau à $180^{\circ} \mathrm{C}$. : $11,6 \% \mathrm{~N}$. et $26,4 \% \mathrm{Cl}$.

en présence d'eau à $61^{\circ} \mathrm{C}$. : $10,3 \% \mathrm{~N}$. et $21,6 \% \mathrm{Cl}$.

en présence d'hydroxyde de potassium : $13,4 \% \mathrm{~N}$. et $16,1 \% \mathrm{Cl}$.

en présence d'éthanol : $11,5 \% \mathrm{~N}$. et $18,6 \% \mathrm{Cl}$.

Tous ces produits sont plus riches en chlore que ceux qui se produisent quand le lait est traité par le chlore gazeux.

Le filtrat a été examiné séparément, et comparé au sérum obtenu sur le même lait par l'action de l'acide acétique :

\begin{tabular}{|c|c|c|}
\hline Dans $100 \mathrm{ce}$ & Sérum par l'acide acétique & Sérum par le chlore \\
\hline Extrait ...... & $5,706 \mathrm{gr}$. & $5,918 \mathrm{gr}$. \\
\hline Cendres... & $0,679 \mathrm{gr}$. & $0,766 \mathrm{gr}$. \\
\hline Lactose (polarisation). & $4,880 \mathrm{gr}$. & $4,260 \mathrm{gr}$. \\
\hline Lactose (Fehling) . . . . . . . & - & $4,182 \mathrm{gr}$. \\
\hline Acidité (phénolphtaléine) ... & $18,2 \mathrm{cc} \cdot \mathrm{n} / 10$ & $47,8 \mathrm{cc} \cdot t^{\mathrm{n}} / 10$ \\
\hline Chlore ............... & $0,087 \mathrm{gr}$. & $1,741 \mathrm{gr}$. \\
\hline Chlore dans les cendres... & $0,075 \mathrm{gr}$. & $0,177 \mathrm{gr}$. \\
\hline
\end{tabular}

(1) Journ. Roy. Army Med. Curps, 1925, 44, 116.

(2) Oxygène actif $=$ chlore actif $\times 0,225$.

(3) Chlorocaseine, Rec. trav. chim. Pays-Bas, 1926, 45, 825. 
Les teneurs en extrait, cendres et chlore augmentent. La quantité de lactose diminue et l'acidité croît. La teneur en chlore dans le sérum par le chlore eomporte une augmentation de $1.741-0,087=1.654$ gr., et pour les cendres de $0,177-0,075=0,120$ gr. La majeure partie du chlore est ainsi volatilisée au cours de l'incinération.

\section{B. Action du brome.}

Le lait écrémé traité par le brome coagule rapidement; la partie coagulée est constituée par de la bromocaséine, contenant 12,1\% d'azote et $21,3 \%$ de brome :

Analyse du précipité après filtration, lavage et dessication : substance : 0 gr., 1734 ; azote (Kjeldahl) : 0 gr., 0210 ou 12,1\%. Substance : 0 gr., 0263 ; brome (ter Meulen) : 0 gr., $0056^{\prime}$ ou $21,3 \%$.

Hopkins et Pinkus (1) ont obtenu aux dépens de la caséine une combinaison bromée contenant $11,17 \%$ de brome, plus pauvre done en brome. Par le traitement de la caséine par le brome en présence de tetrachlorure de carbone j'ai obtenu une bromocaséine (2) contenant $9,6 \%$ d'azote et $35 \%$ de brome ; au cours de l'étude de cette combinaison j'ai préparé ainsi les bromocaséines suivantes :

dans un milieu de tétrachlorure de carbone : 9,6\% $\mathrm{N}$. et $35 \% \mathrm{Br}$.

en présence d'eau à $18^{\circ} \mathrm{C}$. : $12,7 \% \mathrm{~N}$. et $11,6 \% \mathrm{Br}$.

en présence d'eau à $61^{\circ} \mathrm{C}$ : : $11,2 \% \mathrm{~N}$. et $9,7 \% \mathrm{Br}$.

en présence d'hydroxyde de sodium : $14,2 \% \mathrm{~N}$. et $8,1 \% \mathrm{Br}$.

en présence d'ethanol : $12,2 \% \mathrm{~N}$. et $14,7 \% \mathrm{Br}$.

L filtrat séparé du présipité bromé présente les caractères suivants, comparés avec ceux que donne l'acide acétique :

\begin{tabular}{|c|c|c|}
\hline Dans $100 \mathrm{ce}$. & Sérum par l'acide acétique & Sérum par le brome \\
\hline Extrait & $5,706 \mathrm{gr}$. & $6,496 \mathrm{gr}$. \\
\hline Cendres .............. & $0,679 \mathrm{gr}$. & $0,801 \mathrm{gr}$. \\
\hline Lactose (polarisation).... & $4,880 \mathrm{gr}$. & $2,660 \mathrm{gr}$. \\
\hline Lactose (Fehling) & - & $2,430 \mathrm{gr}$. \\
\hline Acidité (phénolphtaléine) & .. 18,2 ec. $n / 10$ & $49,8 \mathrm{cc} . \mathrm{n} / 10$ \\
\hline Chilore .............. & 0,087 gr. & $0,087 \mathrm{gr}$. \\
\hline Chlore dans les cendres. & 0,075 gr. & $0,075 \mathrm{gr}$. \\
\hline Brome................... & - & $3,112 \mathrm{gr}$. \\
\hline Brome dans les cendres & $\ldots$ & $0,200 \mathrm{gr}$. \\
\hline
\end{tabular}

De même que par le traitement par le chlore, le brome provoque une augmentation de la teneur en extrait, cendres et halogènes. La teneur en lactose est notablement diminuée, plus que par le traitement par le chlore. Une partie du brome est fixée dans les cendres.

(1) Ber. deut. chem. Ges., 1898, 31, 1311.

(2) Bromocaséine, Rec. trav. chim. Pays-Bas, 1925, 44, 900. 


\section{Action de l'iode.}

L'action de l'iode sur la caséine a fait l'objet de plusieurs recherches; on a obtenu ainsi des iodocaséines contenant de 7 à $7,5 \%$ d'iode (1), $5,7 \%$ d'iode (2), de 11,3 à $13,5 \%$ d'iode (3). Hofmeister pense (4) que l'iode est fixé dans le noyau cyclique de la molécule; en effet, la tyrosine se combine facilement avec l'iode et forme des combinaisons mono et biiodées, et la décomposition de l'iodocaséine donne naissance à de la diiodotyrosine. Selon Pauly (5) la plus grande partie de l'iode est combinée de manière labile. BLum et Strauss (6) pensent qu'une partie de l'iode est labilement combinée et facile à éliminer par l'acide sulfureux, tandis qu'une autre partie est solidement fixée à un noyau imidazol. LIEBEN et LASzLo (7) ont étudié la question à la lumière de la chimie colloïdale; ils ont fait agir à froid une solutiond'iode ${ }^{\mathrm{n}} / 10$ sur de la caséine dissoute dans de l'hydroxyde de sodium. Après dialyse, le protide restant est précipité par l'acide acétique, et dans le précipité il ne se trouve que $4 \%$ d'iode. L'iode est absorbé jusque $20,4 \%$ par la caséine, mais peut en être extrait par l'alcool presque complètement. LIEBEN et LASZLo concluent de leurs constatations qu'il s'agit essentiellement d'un phénomène d'adsorption.

Par l'action directe de l'iode dissous dans le tétrachlorure de carbone, je n'ai pu obtenir de l'iodocaséine. En faisant agir de l'iode en poudre sur du lait dans lequel la caséine se trouve à l'état naturel, sans ajouter de dissolvant pour éviter les réactions secondaires, j'ai constaté qu'au bout de quelques jours l'iode était combiné; après addition de $0 \mathrm{gr}$., 25 d'iode à $100 \mathrm{~cm}^{3}$ de lait cru, le lait avait repris sa couleur initiale. En répétant les additions d'iode, le lait a coagulé à température ordinaire après introduction totale de $1 \mathrm{gr}$. d'iode pulvérulent dans $100 \mathrm{~cm}^{3} \mathrm{de}$ lait. A la température de $61^{\circ} \mathrm{C}$., $0 \mathrm{gr}$., 5 de poudre d'iode provoquent la coagulation de $100 \mathrm{~cm}^{3}$ de lait après 5 heures, et l'iode est complètement absorbé au bout de 24 heures. Il va sans dire qu'il faut fréquemment agiter les récipients dans lesquel son fait agir la poudre d'iode sur le lait.

De petites quantités d'iode ne sont pas susceptibles de stériliser complètement le lait. Si on traite le lait par de l'iode, il se produit une coagulation au bout de 6 semaines pour des concentrations de 0,09 et de 0 gr., 10 d'iode dans $100 \mathrm{~cm}^{3}$ de lait; après 1 an j'ai trouvé pour 0 gr., 08 d'iode, dans $100 \mathrm{~cm}^{3}$ de lait non coagulé, une acidité de $5,1 \mathrm{~cm}^{3}$ $\mathrm{n} / 10$ pour $10 \mathrm{~cm}^{3}$ de lait. Les résultats de l'examen bactériologique

(1) Blum, Zeitschr. physi,l. Chem., 1898, 26, 298.

(2) Liebrecht, Ber. deut. chem. Ges. 30, 1824.

(3) Oswald, Hofmeister Beiträge 1903, 3, 514.

(4) Zeitschr, physiol. Chem., 24, 159.

(5) Ber. deut. chem. Ges, , 43, 2243.

(6) Zeitschr., physiol. chem., 1920, 112, 111.

(7) Biochem. Zeitschr., 1925, 159, 110. 
étaient indécis et variables. Il serait intéressant de poursuivre cette étude tant au point de vue de la stérilisation du lait que de l'introduction d'iode dans une combinaison organique, chose intéressante au point de vue thérapeutique.

J'ai étudié les variations chimiques produites par la poudre d'iode, après 5,10 et 15 jours, en ajoutant périodiquement l'iode et en secouant fréquemment. Après 5 jours j'avais introduit 0,5 gr. d'iode dans $100 \mathrm{~cm}^{3}$, après 10 jours une $2^{\mathrm{e}}$ quantité de 0 gr., 25 et après 15 jours une $3^{\mathrm{e}}$ quantité de 0 gr., 25 d'iode pour $100 \mathrm{~cm}^{3}$ de lait. Le lait n'était pas coagulé. Les dosages ont été effectués d'après les méthodes habituelles : lé lactose par polarisation, la caséine par précipitation acétique, l'acidité par alcalimétrie sans et après traitement par du sulfite neutre de sodium, la concentration en ions d'après la méthode que j'ai décrite antérieurement (1), en précipitant par de l'alcool et une trace de chlorure de baryumeten employant les colorants de Michaelis, Tous les résultats des tableaux suivants sont exprimés par rapport à $100 \mathrm{cc}$. Il est

Action de l'iode sur le lait entier.

\begin{tabular}{|c|c|c|c|c|c|c|c|c|c|c|c|c|}
\hline \multirow{2}{*}{$\begin{array}{l}\text { Dans } 100 \mathrm{cc} . \\
\text { aprèz jours }\end{array}$} & \multicolumn{3}{|c|}{$1^{\text {ro }}$ série } & \multicolumn{5}{|c|}{$2^{\mathrm{e}}$ série } & \multicolumn{4}{|c|}{$3^{e}$ série } \\
\hline & 0 & 5 & 10 & 5 & 0 & 5 & 10 & 15 & 0 & 5 & 10 & 15 \\
\hline r. & 11.76 & 12.03 & 12.36 & 12.41 & 11.28 & 11.59 & 11.67 & 11.81 & 11.93 & 12.00 & 12 & 12.34 \\
\hline r. eendres & 68 & .70 & 0.71 & & 0.61 & 0.63 & 32 & 70 & .68 & .71 & 71 & 0.72 \\
\hline r. lactose & 56 & 4.56 & .56 & 6 & 4.75 & 4.75 & 4.69 & 5 & 4.56 & 4.63 & 38 & 4.63 \\
\hline rais & & 6 & 5 & & 2.76 & 2.74 & 2.67 & 2.65 & 33 & 3.22 & 40 & 3.28 \\
\hline Gr. caséine & 2.80 & 3.06 & 3.17 & 3.36 & 2.98 & 3.54 & 3.49 & 3.55 & 2.92 & 3.18 & 3.23 & 3.50 \\
\hline $\mathrm{Cm}^{3}$ acidité $\mathrm{n} / 10$ & 15 & 59 & 63 & 63 & 16 & 46 & 64 & 81 & 15 & 51 & 66 & 82 \\
\hline $\mathrm{m}^{3}$ acidité $\mathrm{n}$ & & & & & & & & & & & & \\
\hline & 15 & 50 & 54 & 59 & 16 & 45 & 61 & 79 & 15 & 50 & 62 & 80 \\
\hline $\mathrm{pH}$ & 一 & - & - & - & 5.7 & 5,3 & 4.9 & 4.9 & 6.0 & 5,5 & 5,5 & 4,5 \\
\hline
\end{tabular}

facile de constater que l'augmentation porte sur les teneurs en extrait, cendres, caséine et acidité ; l'iode est combiné aux matières minérales et à la caséine. J'ai examiné de plus près cette combinaison avec la caséine; après avoir traité $100 \mathrm{~cm}^{3}$ de lait centrifugé successivement par 0,5 gr., $0,25 \mathrm{gr}$. et $0,25 \mathrm{gr}$. de poudre d'iode, j'ai, après 6 semaines, séparé la caséine coagulée par filtration, et après lavage avec une

Action de l'iode sur le lait centrifugé.

\begin{tabular}{|c|c|c|c|c|c|c|c|c|c|c|c|c|}
\hline Dan & & $1^{\text {re }} \varepsilon$ & érie & & & $2^{e}$ s & & & & $3^{e} 8$ & irie & \\
\hline après jo & 0 & 5 & 10 & 15 & 0 & 5 & 10 & 15 & 0 & 5 & 10 & 15 \\
\hline Gr. extr & 79 & .66 & 10,08 & 10.16 & 8.46 & 8.95 & 9.19 & 9.20 & 9.0 & .12 & 9.67 & .82 \\
\hline Gr. cendres & 0.65 & 0.66 & 0.67 & 0.71 & 0.62 & 0.62 & 0.63 & 0.68 & 0.71 & 0.72 & 0.78 & .79 \\
\hline Gr. laetose & 4.56 & 4.56 & 4.56 & 4.56 & 4.18 & 4.18 & 4.18 & 4.18 & 4.69 & 4.72 & 4.69 & .69 \\
\hline Gr. caséine & 3.01 & 3.14 & 3.22 & 3.65 & 2.88 & 2.93 & 2.95 & 3.15 & 3.21 & 3.43 & 3.56 & 3.62 \\
\hline Ce. acidité $\mathrm{n} / 10$ & 17 & 68 & 69 & 75 & 17 & 48 & 65 & 86 & 18 & 56 & 68 & 88 \\
\hline $\begin{array}{l}\text { idité } \mathrm{n} / \mathrm{10} \\
\text { sulfite) }\end{array}$ & 1 & & & & 17 & 48 & 3 & 84 & 8 & 50 & 8 & \\
\hline $\mathrm{H}$ & - & - & - & - & 5.5 & 4.9 & 4.8 & 4.8 & 5.9 & 5.4 & 4.7 & \\
\hline
\end{tabular}

(1) Les indicateurs et l'acidité réelle du lait. Le lait, 1927, 7, 140. 
solution de sulfite, avec de l'eau, de l'alcool et de l'éther, puis après dessication, j'ai analysé la poudre jaunâtre obtenue ; c'est une caséine iodée contenant $12,14 \%$ d'azote et $19,8 \%$ d'iode.

Analyse : substance : 0 gr., 2017; azote (Kjeldahl) : 0 gr., $0245 \mathrm{~N}$, soit $12,14 \%$. Substance : 0 gr., 0321 ; iode (ter Meulen) : 0 gr., 00635 , soit $19,8 \%$.

Cette iodocaséine contient la même quantité d'iode que celle de LIEBEN et LASZLO obtenue par l'action d'une solution d'iode sur une solution alcaline de caséine; je n'y trouve pas les caractères de labilité signalés par ces auteurs.

\section{Action des halogènes sur le lactose.}

Les recherches concernant l'action des halogènes sur le lait montrent que le lactose subit une diminution notable de la part du chlore et du brome; l'iode semble sans influence. J'ai sur des solutions de lactose à 10 G.V. $\%$, donnant après stabilisation du pouvoir rotatoire un degré saccharimétrique de $47^{\circ} 5$ (Laurent, tube de $20 \mathrm{~cm}$ ) fait agir le chlore gazeux, le brome liquide et l'iode solide; j'ai employé dans chacun des cas $150 \mathrm{~cm}^{3}$ de solution de lactose, maintenue à froid.

Avec le chlore gazeux, la polarisation diminue rapidement; $j$ 'ai maintenu du chlore en faisant passer du chlore, chaque fois qu'il était combiné ét que le liquide devenait brun; le degré saccharimétrique a passé à +6 , puis à $-6,-10$, pour se relever à $-3,5$ et se stabiliser à $-1^{\circ}$. Au bout de plusieurs semaines, il s'était produit une poudre cristalline insoluble que j'ai séparé par filtration; j'ai obtenu ainsi $2 \mathrm{gr}$. de substance blanche, que j'ai purifiée par cristallisation au moyen de $250 \mathrm{~cm}^{3}$ d'eau bouillante et j'ai recueilli alors 1 gr., 1 d'un produit cristallisé blanc. Il y avait lieu de supposer qu'il s'était formé de l'acide lactobionique $\mathrm{C}_{22} \mathrm{H}_{22} \mathrm{O}_{12}$ ou de l'acide mucique $\mathrm{C}_{6} \mathrm{H}_{10} \mathrm{O}_{8}$. J'ai dissous 0 gr., 0549 de substance dans une quantité suffisante d'eau pour permettre la solution complète, et j'ai titré par la soude $n / 10$ en présence de phénolphtaléine; j'ai employé ainsi $5 \mathrm{~cm}^{3}, 1 \mathrm{n} / 10$. En outre le point de fusion est $210^{\circ} \mathrm{C}$. Ces données concordent parfaitement avec celles que doit fournir l'acide mucique : calculé pour $\mathrm{C}_{6} \mathrm{H}_{10} \mathrm{O}_{3}=210,0$ gr., 0549 d'acide mucique sont neutralisés par $5,2 \mathrm{~cm}^{3} \mathrm{n} / 10$; le point de fusion est $210^{\circ} \mathrm{C}$. Le produit obtenu est done de l'acide mucique.

Avec le brome, la polarisation a diminué également pour atteindre +4 après 5 mois ; il se produit sans doute aussi de l'acide mueique, mais je n'ai pas pu recueillir de produit cristallin insoluble. Dans le cas de l'iode, le pouvoir rotatoire ne s'était abaissé, après 5 mois, que de 47,5 à 46,5 degrés saccharimétriques ; le lactose était donc resté sensiblement intact. 